\title{
Poverty Indices Revisited
}

\author{
Eugene Kouassi ${ }^{1}$, Pierre Mendy ${ }^{2}$, Diaraf Seck ${ }^{2}$, Kern O. Kymn ${ }^{3}$ \\ ${ }^{1}$ Resource Economics, West Virginia University, Morgantown, USA \\ ${ }^{2}$ Faculty of Economics, University of Cheick Anta Diop (UCAD), Dakar, Senegal \\ ${ }^{3}$ Division of Finance and Economics, West Virginia University, Morgantown, USA \\ E-mail: kern.kymn@mail.wvu.edu
}

Received September 13, 2011; revised October 19, 2011; accepted October 28, 2011

\begin{abstract}
In this paper, a new optimization-based approach to constructing a poverty index is considered. From a general perspective, first and second order conditions based on a general poverty intensity function are derived. Then using specific intensity functions defined by $[1,3]$ respectively, we specify related necessary and sufficient conditions and the underlying poverty indices. An extension based on a large class of intensity function is also investigated.
\end{abstract}

Keywords: A General Poverty Index, Necessary and Sufficient Conditions, Other Poverty Indices, Poverty Intensity Functions

\section{Introduction}

How poverty is measured is a central topic in economic and policy analyses. This paper contributes to the literature on this topic by providing methods for measuring poverty in a static environment. In particular, one can define a general poverty index and show that the existing ones are some special cases of a more general index. An extension is also proposed.

The remainder of the paper is organized as follows: a general approach to constructing a poverty index is considered in Section 2. In particular; necessary as well as sufficient conditions to determine the number of poor persons are derived. In Section 3 based on specific intensity functions, conditions to determine the number of poor persons are specified. In Section 4, an extension based on a more general poverty intensity function is proposed. Finally, some concluding remarks are presented in Section 5 .

\section{A General Approach to Construct A Poverty Index}

\subsection{The Problem}

In general poverty issue can be seen as an optimization problem of the so called average intensity poverty function defined as $\Phi():. \mathbb{R}_{+}^{Q} \rightarrow \mathbb{R}$. Specifically, the problem is to minimize a constrained program,

$$
\left\{\begin{array}{l}
\operatorname{Min} \Phi(Y) \\
\text { s.t } \sum_{i=1}^{Q} Y_{i}^{2}=r
\end{array}\right.
$$

where $\sqrt{r}$ is a given strictly positive value which represents the level richness of the $Q$ individuals and $Y_{i}$ is the income of individual $i$. The above minimization program is also equivalent to,

$$
\left\{\begin{array}{l}
\operatorname{Min} \ell(Y, \lambda) \\
\text { s.t }(Y, \lambda) \in \mathbb{R}_{+}^{* Q} \times \mathbb{R}
\end{array}\right.
$$

where $\ell$ represents the Lagrangian and $\lambda$ the Lagrange multipliers. Equation (2) can be solved to determine the number of poor persons, $Q$.

\subsection{Solution}

To solve the above minimization program, first and second order conditions are required.

Theorem 1 Necessary Condition

A necessary condition to get a critical point to problem (1) or (2) is that,

$$
Y_{i}= \pm \sqrt{r} \frac{\frac{\partial \Phi(Y)}{\partial Y_{i}}}{\sqrt{\sum_{i=1}^{Q}\left(\frac{\partial \Phi(Y)}{\partial Y_{i}}\right)^{2}}}, \quad i=1, \cdots, Q
$$

Proof: 
The system of equations is $\frac{\partial \ell(., .)}{\partial Y_{i}}=0, \forall i$; and $\frac{\partial \ell(., .)}{\partial \lambda}=0$, which is equivalent to

$$
\frac{\partial \Phi(Y)}{\partial Y_{\mathrm{i}}}-2 \lambda Y_{\mathrm{i}}=0 ; \mathrm{i}=1, \cdots, Q
$$

and $\sum_{\mathrm{i}=1}^{Q} Y_{\mathrm{i}}^{2}=r$. From the first equation one gets,

$$
\frac{\partial \Phi(Y)}{\partial Y_{\mathrm{i}}}-2 \lambda Y_{\mathrm{i}}=0, \forall \mathrm{i} \Rightarrow\left(\frac{\partial \Phi(Y)}{\partial Y_{\mathrm{i}}}\right)^{2}=4 \lambda^{2} Y_{\mathrm{i}}^{2}
$$

Summing over i , one gets,

$$
4 \lambda^{2} \sum_{\mathrm{i}=1}^{Q} Y_{\mathrm{i}}^{2}=\sum_{\mathrm{i}=1}^{Q}\left(\frac{\partial \Phi(Y)}{\partial Y_{\mathrm{i}}}\right)^{2} .
$$

Hence, $\lambda= \pm \frac{1}{2 \sqrt{r}} \sqrt{\sum_{i=1}^{Q}\left(\frac{\partial \Phi(Y)}{\partial Y_{i}}\right)^{2}}$

\section{Theorem 2 Sufficient Condition}

Let $\Phi$ and $g$ be two functions of class $C^{2}$ where $\Phi$ is the poverty intensity function and

$g(Y)=\sum_{\mathrm{i}=1}^{Q} Y_{\mathrm{i}}^{2}-r$. Suppose that $\mathrm{A}$ is defined such that $A=\left(a_{\mathrm{i}}\right), \mathrm{i}=1, \cdots, Q$, where

$$
a_{\mathrm{i}}= \pm \sqrt{r} \frac{\frac{\partial \Phi(Y)}{\partial Y_{\mathrm{i}}}}{\sqrt{\sum_{\mathrm{i}=1}^{Q}\left(\frac{\partial \Phi(Y)}{\partial Y_{\mathrm{i}}}\right)^{2}}}
$$

Assume that $\nabla g(A) \neq 0$, then, if

$$
(-1) \operatorname{det} M_{\mathrm{i}}(A)>0, \mathrm{i}=2, \cdots, Q
$$

i.e., if all determinants of the bordered principal minors $M_{\mathrm{i}}(A)$ are negative, then $\Phi$ has a strict minimum

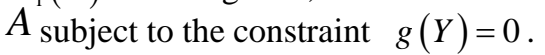

Proof: (A general proof is given in Proposition 3)

Since one is dealing with a constrained optimization problem, one can consider the matrix of bordered principal minors $M_{\mathrm{i}}(A)$ of $A$ which is defined as,

$$
M_{\mathrm{i}}(A)=\left(\begin{array}{cccc}
\frac{\partial^{2} \ell(A)}{\partial^{2} Y_{1}^{2}(A)} & \cdots & \frac{\partial^{2} \ell(A)}{\partial Y_{\mathrm{i}} \partial Y_{1}(A)} & \frac{\partial g(A)}{\partial Y_{1}(A)} \\
\vdots & \ddots & \vdots & \vdots \\
\frac{\partial^{2} \ell(A)}{\partial Y_{1} \partial Y_{\mathrm{i}}(A)} & \cdots & \frac{\partial^{2} \ell(A)}{\partial^{2} Y_{\mathrm{i}}^{2}(A)} & \frac{\partial g(A)}{\partial Y_{\mathrm{i}}(A)} \\
\frac{\partial g(A)}{\partial Y_{1}(A)} & \cdots & \frac{\partial g(A)}{\partial Y_{\mathrm{i}}(A)} & 0
\end{array}\right)
$$

and therefore,

$$
M(A)=\left(\begin{array}{cccc}
\frac{\partial^{2} \ell(A)}{\partial^{2} Y_{1}^{2}(A)} & \cdots & \frac{\partial^{2} \ell(A)}{\partial Y_{Q} \partial Y_{1}(A)} & \frac{\partial g(A)}{\partial Y_{1}(A)} \\
\vdots & \ddots & \vdots & \vdots \\
\frac{\partial^{2} \ell(A)}{\partial Y_{1} \partial Y_{Q}(A)} & \cdots & \frac{\partial^{2} \ell(A)}{\partial^{2} Y_{Q}^{2}(A)} & \frac{\partial g(A)}{\partial Y_{Q}(A)} \\
\frac{\partial g(A)}{\partial Y_{1}(A)} & \cdots & \frac{\partial g(A)}{\partial Y_{Q}(A)} & 0
\end{array}\right)
$$

One can then deduce the following proposition:

\section{Proposition 3}

Under the assumptions of Theorem 2, if

$$
\sum_{k=1}^{Q} \sum_{r=1}^{Q} \frac{\partial^{2} \ell}{\partial Y_{k} \partial Y_{r}}(A)=h_{r} h_{k}>0
$$

$\forall H=\left(h_{1}, \cdots, h_{Q}\right)^{t} \in \mathbb{R}^{Q}$ such that $H \neq 0$ and satisfying $\nabla g(A) \cdot H=0$, then $A$ is a strict local minimum of $\Phi(.,$.$) subject to constraint g(Y)=0$.

\section{Proof:}

One just needs to show that there exists a neighborhood $U_{A}$ such that $X \in U_{A} \cap S$ and $X \neq A$, $\ell(X)>\ell(A)$, with $S=\left\{X \in \mathbb{R}^{Q} / g(X)=0\right\}$.

Let $V_{A}$ be a neighborhood of $A$. Let $X \in V_{A} \cap S$ and let $H \in \mathbb{R}^{Q}$ such that $\nabla g(A) H=0$. Then,

$$
\begin{aligned}
\ell & (X)-\ell(A) \\
= & \frac{1}{2} \sum_{k=1}^{Q} \sum_{r=1}^{Q} \frac{\partial^{2} \ell(A)}{\partial X_{k} \partial X_{r}}\left(X_{k}-a_{k}\right)\left(X_{r}-a_{r}\right) \\
& +o\left(\|X-A\|^{2}\right)
\end{aligned}
$$

Set $H=X-A$. It then follows that,

$$
\begin{aligned}
& \ell(H+A)-\ell(A)= \\
& \frac{1}{2} \sum_{k=1}^{Q} \sum_{r=1}^{Q} \frac{\partial^{2} \ell(A)}{\partial X_{k} \partial X_{r}} h_{k} h_{r}+o\left(\|H\|^{2}\right)
\end{aligned}
$$

For $H$ such that $H \neq 0$ and $H+A \in V_{A} \cap S$, set

$$
Q(H)=\sum_{k=1}^{Q} \sum_{r=1}^{Q} \frac{\partial^{2} \ell(A)}{\partial X_{k} \partial X_{r}} h_{k} h_{r} .
$$

$Q$ is a continuous function on $V_{A} \cap S$. Assuming $K$ is a closed of non empty interior and bounded set; $K \subset V_{A} \cap S$. Then, $Q$ reaches its minimum value on $K$ and since

$$
\begin{aligned}
& \sum_{k=1}^{Q} \sum_{r=1}^{Q} \frac{\partial^{2} \ell(A)}{\partial X_{k} \partial X_{r}} h_{k} h_{r}>0, \quad \exists q>0: Q(H) \geq q\|H\|^{2} . \\
& \text { Hence, } \begin{array}{l}
\ell(H+A)-\ell(A) \geq q\|H\|^{2}(1+o(1)) \\
\Rightarrow \ell(H+A)>\ell(A)
\end{array}
\end{aligned}
$$


By continuity of $Q$, there exists a neighborhood $U_{A} \subset V_{A}$ such that: $\forall X \in U_{A} \cap S, \ell(X)>\ell(A)$.

Next, one can specify first and second order conditions based on three well known poverty intensity functions and thereafter obtain the expressions for the underlying poverty indices.

\section{Specific Poverty Indices}

\subsection{The Problem}

Consider the first and second order conditions in three main specific average intensity functions and assume that the concern is the condition for a given individual to be poor.

\subsection{Sen's Solution (1976)}

A class of intensity function considered by Sen [1] is,

$$
\Phi(Y)=\frac{2}{H(Q+1)} \sum_{j=1}^{Q}(Q-j+1) \frac{\left(Z-Y_{j}\right)}{Z}
$$

where $H \in\{N, Q\}, Z$ is the poverty line, $Y_{j}$ is the revenue of the poor $j, Q$ is the number of poor persons and $N$ is the size of the population. This function is convex and therefore Theorem 2 is a sufficient condition for a minimum. Solving, one gets,

$$
Y_{\mathrm{i}}=\sqrt{r} \frac{(Q-\mathrm{i}+1)}{\sqrt{\sum_{j=1}^{Q}(Q-j+1)^{2}}}, \mathrm{i}=1, \cdots, Q
$$

\section{Proposition 4}

Consider [1]'s average intensity function. Then, the number of poor persons in a population of $N$ individuals given a revenue constraint is obtained as,

$$
\begin{gathered}
\sqrt{r} \frac{(Q-\mathrm{i}+1)}{Z \sqrt{\sum_{j=1}^{Q}(Q-j+1)^{2}}} \leq 1, \\
\mathrm{i}=1, \cdots, Q ; Q=1, \cdots, N
\end{gathered}
$$

Proof: (Straightforward)

\subsection{Forster, Greer and Thorbecke's (FGT) Solution (1984)}

[2] propose the following average intensity function,

$$
\Phi(Y)=\frac{1}{H} \sum_{j=1}^{Q}\left(\frac{Z-Y_{j}}{Z}\right)^{\alpha}
$$

where $H \in\{N, Q\}, \alpha>0$ and the other parameters are defined as above. It is easy to verify that for $\alpha>1$, the [2] average intensity function is strictly convex while for $0<\alpha<1$, the underlying quadratic form is negative definite and therefore the [2] average intensity function is strictly concave. The [2] result is obtained as follows, $\forall T, Y \in \mathbb{R}^{Q}$

$$
\begin{aligned}
& \left(\nabla^{2} \Phi(Y)(T-Y),(T-Y)\right) \\
& =\frac{1}{H} \alpha(\alpha-1) \sum_{\mathrm{i}=1}^{Q}\left(\frac{Z-Y_{i}}{Z}\right)^{\alpha-2}\left(T_{\mathrm{i}}-Y_{\mathrm{i}}\right)^{2}
\end{aligned}
$$

A necessary condition to get a critical vector point is given by Theorem 2, i.e.,

$$
Y_{\mathrm{i}}=\sqrt{r} \frac{\left(Z-Y_{\mathrm{i}}\right)^{\alpha-1}}{\sqrt{\sum_{j=1}^{Q}\left(Z-Y_{j}\right)^{2 \alpha-2}}}, \mathrm{i}=1, \cdots, Q
$$

In case where $\alpha \geq 1$, the above critical vector point becomes a minimum for function $\Phi(.,$.$) . When \alpha \in(0,1)$, the average intensity function $\Phi(.,$.$) is strictly concave$ and the critical vector point $Y_{\mathrm{i}}$ is a maximum. Therefore, one gets:

\section{Proposition 5}

Consider [2]'s average intensity function. Then, the number of poor persons in a population of $N$ individuals given a revenue constraint is obtained as,

$$
Y_{\mathrm{i}}=\frac{r}{Q}
$$

Proof: (Straightforward by introducing the Lagrangian and based on the constraint $\sum_{\mathrm{i}=1}^{Q} Y_{\mathrm{i}}=r$ ).

\subsection{Shorrock's Solution (1995)}

[3] considers the following average intensity function,

$$
\Phi(Y)=\frac{1}{H^{2}} \sum_{j=1}^{Q}(2 N-2 j+1) \frac{\left(Z-Y_{j}\right)}{Z}
$$

where $H \in\{N, Q\}$ and the other parameters are defined as above. Since this function is convex, Theorem 2 is a sufficient condition for a minimum. One gets,

$$
\frac{\partial \Phi(Y)}{\partial Y_{\mathrm{i}}}=-\frac{1}{H^{2} Z}(2 N-2 \mathrm{i}+1)
$$

Using Theorem 2, one immediately gets

$$
Y_{\mathrm{i}}= \pm \sqrt{r} \frac{(2 N-2 \mathrm{i}+1)}{\sqrt{\sum_{j=1}^{Q}(2 N-2 j+1)^{2}}}, \mathrm{i}=1, \cdots, Q
$$

Since $Y_{\mathrm{i}} \geq 0$, one obtains,

$$
Y_{\mathrm{i}}=\sqrt{r} \frac{(2 N-2 \mathrm{i}+1)}{\sqrt{\sum_{j=1}^{Q}(2 N-2 j+1)^{2}}}, \mathrm{i}=1, \cdots, Q
$$

\section{Proposition 6}


Consider [3]'s average intensity function. Then, the number of poor persons in a population of $N$ individuals given a revenue constraint is obtained as,

$$
\begin{aligned}
& \qquad \begin{array}{l}
\sqrt{r} \frac{(2 N-2 \mathrm{i}+1)}{Z \sqrt{\sum_{j=1}^{Q}(2 N-2 j+1)^{2}}} \leq 1, \mathrm{i}=1, \cdots, Q ; \\
Q
\end{array}=1, \cdots, N \\
& \text { Proof: (Straightforward) }
\end{aligned}
$$

\section{An Extension}

Consider $\Phi(.,$.$) a more general average intensity func-$ tion. Assume that $\Phi(. .$.$) is a regular function which$ can be decomposed using a Taylor expansion. Using the vector origin, one can get the Taylor expansion of the above function at any order. Then, using regression techniques, the underlying coefficients can be estimated. In this case, it is important to get a dispersion measure, i.e., the variance which can then be minimized thereafter.

In this paper, for simplicity, one considers Taylor expansion of order 1 only which gives very interesting results.

Specifically, consider an average poverty intensity function which is derivable in $A=(Z, \cdots, Z)$ (vector $A$ has $Q$ columns) and which is such that the derivatives of superior orders are null in $A$. Note that the techniques proposed in this paper can be used only in the neighborhood of the origin. If this condition is not satisfied, one can always use an appropriate change of variable to get a Taylor expansion in the neighborhood of the origin $0=(0, \cdots, 0)$. The general intensity function considered is,

$$
\Phi(Y)=\frac{1}{\delta(H)} \sum_{j=1}^{Q} f(N, j, H) \gamma\left(\frac{Z-Y_{j}}{Z}\right)
$$

where $H \in\{Q, N\}, \delta, f$ and $\gamma$ are some given functions and the other parameters are defined as previously. Its Taylor expansion is,

$$
\begin{aligned}
\Phi(Y)= & \Phi(0)+\frac{\partial \Phi(0)}{\partial Y_{1}} Y_{1}+\frac{\partial \Phi(0)}{\partial Y_{2}} Y_{2} \\
& +\cdots+\frac{\partial \Phi(0)}{\partial Y_{Q}} Y_{Q}+o(\|Y\|)
\end{aligned}
$$

Set $\varepsilon=o(\|Y\|), \Phi(.,$.$) can be approximated by,$

$$
\begin{gathered}
\Phi(Y) \sim \\
\Phi(0)+\frac{\partial \Phi(0)}{\partial Y_{1}} Y_{1}+\frac{\partial \Phi(0)}{\partial Y_{2}} Y_{2} \\
+\cdots+\frac{\partial \Phi(0)}{\partial Y_{Q}} Y_{Q}
\end{gathered}
$$

By setting $\beta_{\mathrm{i}}=\frac{\partial \Phi(0)}{\partial Y_{\mathrm{i}}}$ and assuming that the minimization of the average intensity function is obtained with a constraint and based on Theorem 2, one gets the following critical vector point,

$$
Y_{\mathrm{i}}= \pm \sqrt{r} \frac{\beta_{i}}{\sqrt{\sum_{j=1}^{Q} \beta_{j}^{2}}}, \mathrm{i}=1, \cdots, Q
$$

Proof:

It suffices to consider the expression derived in equation (22). By replacing the partial derivatives of $\Phi($.$) with respect to \beta_{i}$, one gets a linear expression. Then, using the proof of Theorem 1 , one gets the desired result.

The $\beta_{i}$ 's are known as regression coefficients and have to be estimated using OLS technique where,

$$
Y=\beta\left(1, Y^{t}\right)^{t}, \beta=\left(\beta_{0}, \beta_{1}, \cdots, \beta_{Q}\right)
$$

where $Y^{t}$ is the transpose vector of $Y$. The variance of $Y$ is given by,

$$
V(Y)=\frac{\sigma^{2}}{n}+X^{t}\left(D^{t} D\right)^{-1} X+\sigma^{2}
$$

where $X=(1, D), D=\left(d^{1}, d^{2}, \cdots, d^{Q}\right)$ and $d^{i}(\mathrm{i} \in\{1, \cdots, Q\})$ is a column vector of $n$ components each equal to 1 and $\sigma^{2}=\operatorname{Var}(o\|Y\|)$ is the rest of Taylor expansion which is interpreted as a random variable. This variance is also characterized by $\operatorname{Var}\left(\beta_{\mathrm{i}}\right)=\sigma^{2} d^{\mathrm{ii}}$, with

$$
D^{t} D=\left(d_{\mathrm{ij}}\right)_{\substack{1 \leq i \leq Q \\ 1 \leq j \leq Q}},\left(D^{t} D\right)^{-1}=\left(d^{\mathrm{ij} j}\right)_{\substack{1 \leq \mathrm{i} \leq Q \\ 1 \leq j \leq Q}}
$$

One assumes that

$$
1^{t} . D=0=(0, \cdots, 0)
$$

The uni-row matrix 0 has $Q$ columns. Once the $\beta_{\mathrm{i}}$ 's are obtained, one can get the number of poor persons based on the following condition:

\section{Proposition 7}

Consider the general average intensity function. Then, the number of poor in a population of $N$ individuals given a revenue constraint is given by,

$$
\begin{gathered}
\pm \sqrt{r} \frac{\beta_{\mathrm{i}}}{Z \sqrt{\sum_{j=1}^{Q} \beta_{j}^{2}}} \leq 1, \mathrm{i}=1, \cdots, Q ; \\
Q=1, \cdots, N
\end{gathered}
$$

Proof: (Straightforward).

\section{Final Remarks}

This paper considered a general poverty index and derived the first and second order conditions to get such an 
index. The well known $[1,3]$ indices based on specific intensity functions are then obtained as special cases of this more general poverty index. An extension based on a large class of intensity function is also investigated.

\section{References}

[1] A. K. Sen, "Poverty: An Ordinal Approach to Measurement,” Econometrica, Vol. 44, No. 2, 1976, pp. 219-231.

\section{doi: $10.2307 / 1912718$}

[2] J. Foster, J. Greer and E. Thorbecke, "A Class of Decomposable Poverty Measures,” Econometrica, Vol. 52, No. 3, 1994, pp. 761-765. doi:10.2307/1913475

[3] A. F. Shorrocks, "Revisiting the Sen Poverty Index," Econometrica, Vol. 63, No. 5, 1995, pp. 1225-1230. doi:10.2307/2171728 